\title{
Effects of molecular transport in LES/PDF of piloted turbulent dimethyl ether/air jet flames
}

\author{
Jiaping You ${ }^{\mathrm{a}}$, Yue Yang ${ }^{\mathrm{a}, \mathrm{b}, \mathrm{c}, *}$, Stephen B. Pope ${ }^{\mathrm{d}}$, \\ ${ }^{a}$ State Key Laboratory for Turbulence and Complex Systems, College of Engineering, \\ Peking University, Beijing 100871, China \\ ${ }^{b}$ Center for Applied Physics and Technology, College of Engineering, Peking University, \\ Beijing 100871, China \\ ${ }^{c}$ Beijing Innovation Center for Engineering Science and Advanced Technology, Peking \\ University, Beijing 100871, China \\ ${ }^{d}$ Sibley School of Mechanical and Aerospace Engineering, Cornell University, Ithaca, NY \\ 14853, USA
}

\begin{abstract}
We report large-eddy simulation (LES)/probability density function (PDF) modeling of piloted turbulent dimethyl ether (DME)/air jet flames with a skeletal chemical mechanism. The modeled PDF transport equation with three different implementations of molecular transport in the interaction-byexchange-with-the-mean (IEM) mixing model is solved using the NGA/HPDF code in order to assess the a posteriori performance of these LES/PDF methodologies applied to the DME flames D and F proposed in the TNF Workshop. The three implementations considered are the classical randomwalk model (IEM-RW), the mean-drift model with a single molecular diffusivity (IEM-MD), and the mean-drift model with differential diffusion (IEM-
\end{abstract}

${ }^{*}$ Corresponding author. Address: 310 Building 1, College of Engineering, Peking University, Beijing 100871, China

Email address: yyg@pku.edu.cn (Yue Yang)

Preprint submitted to Combustion and Flame

November 2, 2016

(C) 2016. This manuscript version is made available under the Elsevier user license

http://www.elsevier.com/open-access/userlicense/1.0/ 
DD). Better quantitative agreement with experimental data for the mean and root-mean-square profiles of mixture fraction, temperature and major species mole fractions and conditional means on mixture fraction are obtained with the IEM-MD model than with the IEM-RW model. In LES of the DME flame series, the molecular diffusivity dominates the turbulent diffusivity in the centerline region, so the accurate modeling of molecular transport plays a significant role in LES/PDF of these jet flames. Nevertheless, the IEMDD model shows only minor effects on the model predictions compared to IEM-MD in these DME flames.

Keywords:

Large-eddy simulation, PDF method, Dimethyl ether, Molecular transport, Differential diffusion

\section{1. Introduction}

2 Dimethyl ether (DME) is considered to be promising as an alternative 3 diesel fuel candidate because of its low tendency to form soot, low emission 4 levels of nitrogen oxides, and good auto-ignition performance [1]. It is a chem5 ically more complex fuel than methane and involves a wider range of time 6 scales in its chemical kinetics. Investigations on turbulence-chemistry inter7 actions are crucial for the use of complex fuels such as DME in practical ap8 plications. Considering that piloted turbulent partially-premixed $\mathrm{CH}_{4} /$ air jet 9 flames have been a paradigm for the investigation on turbulence-chemistry 10 interactions, the DME flame series was proposed as new benchmark flames 
for international collaborations on the validation of combustion models owing to the increased complexity of its chemistry (from C1 to C2) [2].

The hybrid LES/PDF method has been proven to be successful in simulations of turbulent combustion owing to its exact treatment of the non-linear chemical source terms [7-10]. In PDF modeling, the closure of the conditional molecular diffusion term, including molecular mixing in composition space and molecular transport in physical space, in the PDF evolution equation is a major challenge. The modeling of mixing is critical in non-premixed combustion as it controls small-scale mixing and subsequent chemical reactions. In contrast, the effect of molecular transport is often neglected in existing Reynolds averaged Navier-Stokes (RANS)/PDF calculations [11, 12], because the magnitude of molecular transport is presumed much smaller than that of turbulent transport in high-Reynolds-number flows. Nevertheless, in the LES context, molecular transport may dominate over the sub grid scale turbulent transport in the regions of high temperature or low turbulent inten- 
sity. Kemenov and Pope [13] demonstrated that molecular transport plays an important role in the further downstream jet development and continues

to be dominant in regions near the centerline. More recently, Wang and Kim [14] showed that in some cases molecular transport can reach the same or even higher order of the magnitude as turbulent transport and affects the robustness of mixing models in RANS/PDF simulations. Therefore, it is necessary to represent molecular transport accurately in LES/PDF studies of turbulent reacting flows.

In the classical IEM model, the effect of molecular transport is incorporated as a random-walk term in the particle transport equation, which is referred to as IEM-RW below. As noted by McDermott and Pope [15], this treatment gives rise to a spurious production of scalar variance in the DNS limit, so an alternative approach was proposed to model molecular transport as a mean-drift term in the scalar equation, and it was then implemented numerically in simple scalar transport cases [16]. This implementation is referred to as IEM-MD below. It is noted that the IEM-MD model can incorporate differential diffusion $[17,18]$ that can have a first-order effect on combustion processes [19-22]. For the DME-D flame, Popp et al. [6] showed that the effects of differential diffusion are not dominant by indirectly incorporating the non-unity-Lewis number into the tabulation of flamelets, which can also be investigated using LES/PDF.

Therefore, the DME flame series, with different Reynolds numbers and local-extinction levels, can serve as a good candidate case to evaluate the ef- 
57 fects of molecular transport and differential diffusion on the predicability of

combustion models. In this paper, the capabilities of different implementa-

59

60

$738 \mathrm{~mm}$ and $18.2 \mathrm{~mm}$, respectively.

Table 1: Bulk velocities and Reynolds numbers of the DME-D and DME-F flames [4].

\begin{tabular}{ccccc}
\hline & $U_{j}[\mathrm{~m} / \mathrm{s}]$ & $U_{\text {pilot }}[\mathrm{m} / \mathrm{s}]$ & $U_{\text {coflow }}[\mathrm{m} / \mathrm{s}]$ & $R e=U_{j} D / \nu$ \\
\hline DME-D & 45.9 & 1.1 & 0.9 & 29,300 \\
DME-F & 91.8 & 2.1 & 0.9 & 58,600 \\
\hline
\end{tabular}




\subsection{LES/PDF modeling}

79 In reacting flows, the composition variable $\phi$ consists of $n_{s}$ species mass

$$
\frac{\partial}{\partial t}(\bar{\rho} \widetilde{f})+\nabla \cdot\left[\bar{\rho}\left(\widetilde{\boldsymbol{u}}+\widetilde{\boldsymbol{u}^{\prime} \mid \boldsymbol{\psi}}\right) \widetilde{f}\right]=-\frac{\partial}{\partial \psi_{\alpha}}\left[\bar{\rho}\left(\frac{1}{\bar{\rho}} \overline{\nabla \cdot\left(\rho \Gamma_{(\alpha)} \nabla \phi_{\alpha}+\rho \phi_{\alpha} \boldsymbol{V}_{c}\right) \mid \boldsymbol{\psi}}+S_{\alpha}(\boldsymbol{\psi})\right) \tilde{f}\right]
$$

91

92

fractions $\boldsymbol{Y}=\left\{Y_{\alpha}, \alpha=1, \ldots, n_{s}\right\}$ and enthalpy $h$. Using the new framework of the self-conditioned PDF [24], we define $\widetilde{f}(\boldsymbol{\psi} ; \boldsymbol{x}, t)$ as the one-point, one-time density-weighted joint PDF of composition conditioned on the resolved velocity $\widetilde{\boldsymbol{u}}$, where $\boldsymbol{\psi}$ denotes the composition sample space for $\boldsymbol{\phi}$. The LES mean composition is $\widetilde{\boldsymbol{\phi}}=\int \boldsymbol{\psi} \widetilde{f} d \boldsymbol{\psi}$ and the LES mean density is $\bar{\rho}=1 / \int \tilde{f} / \rho(\boldsymbol{\psi}) d \boldsymbol{\psi}$, where integration is over the whole composition space. Practically, the self-conditioned framework and the filtering approach [25] for LES/PDF are different only on the conceptual level, and thus do not lead to substantially different partial differential equations to be solved.

The composition PDF method accounts for the evolution of $\widetilde{f}(\boldsymbol{\psi} ; \boldsymbol{x}, t)$ by solving a modeled PDF transport equation [24]

where $\boldsymbol{u}^{\prime}$ is the residual velocity fluctuation, $\Gamma_{(\alpha)}$ is the molecular diffusivity of species $\alpha, S_{\alpha}$ is the chemical reaction source term, $\boldsymbol{V}_{c} \equiv-\sum_{\alpha=1}^{n_{s}} \Gamma_{(\alpha)} \nabla \phi_{\alpha}$ is a correction velocity, which is non-zero only if there is differential diffusion, and 

described in detail below and are summarized in Table 2.

Table 2: Summary of the three different models

\begin{tabular}{lccc}
\hline Model name & IEM-RW & IEM-MD & IEM-DD \\
\hline Model equations & $(4)$ and $(5)$ & $(6)-(8)$ & $(7)-(9)$ \\
Molecular-transport model & Random walk & Mean drift & Mean drift \\
Incorporate differential diffusion & No & No & Yes \\
Satisfy the DNS limit & No & Yes & Yes \\
Relative computational cost & 1.0 & 1.3 & 1.4 \\
\hline
\end{tabular}

$$
\gamma_{\alpha}(\boldsymbol{\psi}, \boldsymbol{x}, t)=-\Omega_{m}\left(\psi_{\alpha}-\widetilde{\phi}_{\alpha}\right)
$$

where $\widetilde{\phi}_{\alpha}$ is the density-weighted mean composition of species $\alpha$ and $\Omega_{m}$ is the mixing rate defined below (Eq. (10)). It is noted that all the LES mean quantities are calculated by the Particle-in-Cell estimation from the particles in each LES cell with a smoothing technique [16], which can reduce statistical noises before taking derivatives of the LES mean quantities.

The transport term $\widetilde{\mathcal{D}}_{\alpha}$ is implemented by three different models that are 
1. In the IEM model with the random-walk implementation (IEM-RW) [28], Eq. (1) with the model Eq. (3) is solved by a particle method

$$
\begin{aligned}
d \boldsymbol{X}^{*}(t) & =\left(\widetilde{\boldsymbol{u}}+\frac{\nabla \bar{\rho}\left(\widetilde{\Gamma}+\widetilde{\Gamma}_{T}\right)}{\bar{\rho}}\right)^{*} d t+\left(2 \widetilde{\Gamma}^{*}+2 \widetilde{\Gamma}_{T}^{*}\right)^{1 / 2} d \boldsymbol{W}, \\
\frac{d \phi_{\alpha}^{*}(t)}{d t} & =-\Omega_{m}^{*}\left(\phi_{\alpha}^{*}-\widetilde{\phi}_{\alpha}^{*}\right)+S_{\alpha}\left(\phi^{*}\right),
\end{aligned}
$$

where $\boldsymbol{X}^{*}(t)$ is the particle position, $\widetilde{\Gamma}_{T}$ is the turbulent diffusivity, $\boldsymbol{W}$ is an isotropic, vector-valued Wiener process and the superscript “*” on mean quantities denotes evaluating those quantities at $\boldsymbol{X}^{*}(t)$ by interpolation. The assumption of unity Lewis number, i.e., $\Gamma_{(\alpha)}=\Gamma$, is made for IEM-RW, where $\Gamma$ is assumed to be equal to the thermal diffusivity of the mixture that is calculated from CHEMKIN's transport library [29]. Note that the transport term $\widetilde{\mathcal{D}}_{\alpha}$ in Eq. (2) is given implicitly by the random-walk model $\left(2 \widetilde{\Gamma}^{*}\right)^{1 / 2} d \boldsymbol{W}$ in Eq. (4).

2. In the IEM model with the mean-drift implementation and the unity Lewis number (IEM-MD) $[15,16]$, the transport term $\widetilde{\mathcal{D}}_{\alpha}$ in Eq. (2) is represented as a mean drift term

$$
\widetilde{\mathcal{D}}_{\alpha} \equiv \frac{1}{\bar{\rho}} \nabla \cdot\left(\bar{\rho} \Gamma \nabla \widetilde{\phi}_{\alpha}\right) .
$$


119

120

Using the particle method, Eq. (1) is solved as

$$
\begin{aligned}
d \boldsymbol{X}^{*}(t) & =\left(\widetilde{\boldsymbol{u}}+\frac{\nabla \bar{\rho} \widetilde{\Gamma}_{T}}{\bar{\rho}}\right)^{*} d t+\left(2 \widetilde{\Gamma}_{T}^{*}\right)^{1 / 2} d \boldsymbol{W}, \\
\frac{d \phi_{\alpha}^{*}(t)}{d t} & =-\Omega_{m}^{*}\left(\phi_{\alpha}^{*}-\widetilde{\phi}_{\alpha}^{*}\right)+\widetilde{\mathcal{D}}_{\alpha}^{*}+S_{\alpha}\left(\phi^{*}\right) .
\end{aligned}
$$

In Eq. (7), the random walk is due solely to the turbulent diffusivity, in contrast to Eq. (4) for IEM-RW. Additionally, a physically motivated lower limit is imposed on the mixing rate $\Omega_{m}^{*}[15]$ to ensure that each composition remains within its own bounds as $\min \left(\phi_{\alpha}^{*}(t)\right) \leq \phi_{\alpha}^{*}(t+$ $\Delta t) \leq \max \left(\phi_{\alpha}^{*}(t)\right)$

3. In the IEM model with the mean-drift implementation and differential diffusion (IEM-DD)[15, 16], differential diffusion is incorporated as

$$
\widetilde{\mathcal{D}}_{\alpha} \equiv \frac{1}{\bar{\rho}} \nabla \cdot\left(\bar{\rho} \Gamma_{(\alpha)} \nabla \widetilde{\phi}_{\alpha}\right)-\frac{1}{\bar{\rho}} \nabla \cdot\left(\bar{\rho} \widetilde{\phi}_{\alpha} \boldsymbol{V}_{c}\right)
$$

The corresponding particle equations are the same as those for IEMMD, but with $\widetilde{\mathcal{D}}_{\alpha}$ defined by Eq. (9). In order to reduce the computational cost, molecular diffusivities are obtained with $L e_{\alpha}$ assumed to be constant throughout the flame, i.e., $\Gamma_{(\alpha)}=\Gamma L e_{\alpha}$, where $L e_{\alpha}$ are obtained in the burnt gas of a one-dimensional, laminar flame with full transport properties, using CHEMKIN [30]. Their values are given in Table 3 .

In all the implementations of the IEM model, the particle composition 
Table 3: Constant species Lewis numbers.

\begin{tabular}{|c|c|c|c|c|c|}
\hline Species & $L e$ & Species & $L e$ & Species & $L e$ \\
\hline $\mathrm{H}$ & 0.16 & $\mathrm{HCO}$ & 1.20 & $\mathrm{CH}_{3} \mathrm{OCH}_{3}$ & 1.65 \\
\hline $\mathrm{H}_{2}$ & 0.27 & $\mathrm{C}_{2} \mathrm{H}_{5}$ & 1.36 & $\mathrm{HOCH}_{2} \mathrm{O}$ & 1.34 \\
\hline $\mathrm{CH}_{2}$ & 0.90 & $\mathrm{CH}_{2} \mathrm{O}$ & 1.21 & $\mathrm{CH}_{3} \mathrm{OCO}$ & 1.80 \\
\hline $\mathrm{CH}_{2}(\mathrm{~S})$ & 0.90 & $\mathrm{C}_{2} \mathrm{H}_{6}$ & 1.37 & $\mathrm{CH}_{3} \mathrm{OCHO}$ & 1.81 \\
\hline $\mathrm{CH}_{3}$ & 0.92 & $\mathrm{CH}_{2} \mathrm{OH}$ & 1.23 & $\mathrm{CH}_{3} \mathrm{OCH}_{2} \mathrm{O}$ & 1.91 \\
\hline $\mathrm{O}$ & 0.66 & $\mathrm{CH}_{3} \mathrm{O}$ & 1.23 & $\mathrm{CH}_{3} \mathrm{OCH}_{2} \mathrm{OH}$ & 1.74 \\
\hline $\mathrm{CH}_{4}$ & 0.93 & $\mathrm{O}_{2}$ & 1.04 & $\mathrm{OCH}_{2} \mathrm{OCHO}$ & 1.79 \\
\hline $\mathrm{OH}$ & 0.67 & $\mathrm{HO}_{2}$ & 1.04 & $\mathrm{HOCH}_{2} \mathrm{OCO}$ & 1.79 \\
\hline $\mathrm{H}_{2} \mathrm{O}$ & 0.79 & $\mathrm{H}_{2} \mathrm{O}_{2}$ & 1.03 & $\mathrm{CH}_{3} \mathrm{OCH}_{2} \mathrm{O}_{2}$ & 1.79 \\
\hline $\mathrm{C}_{2} \mathrm{H}_{2}$ & 1.13 & $\mathrm{CO}_{2}$ & 1.28 & $\mathrm{CH}_{2} \mathrm{OCH}_{2} \mathrm{O}_{2} \mathrm{H}$ & 1.79 \\
\hline $\mathrm{C}_{2} \mathrm{H}_{3}$ & 1.14 & $\mathrm{CH}_{3} \mathrm{HCO}$ & 1.44 & $\mathrm{HO}_{2} \mathrm{CH}_{2} \mathrm{OCHO}$ & 1.83 \\
\hline $\mathrm{CO}$ & 1.03 & $\mathrm{CH}_{3} \mathrm{OCH}_{2}$ & 1.65 & $\mathrm{O}_{2} \mathrm{CH}_{2} \mathrm{OCH}_{2} \mathrm{O}_{2} \mathrm{H}$ & 1.86 \\
\hline $\mathrm{C}_{2} \mathrm{H}_{4}$ & 1.07 & $\mathrm{HCOOH}$ & 1.63 & $\mathrm{~N}_{2}$ & 1.01 \\
\hline
\end{tabular}

$135 \phi^{*}$ relaxes to the mean composition $\tilde{\phi}^{*}$ interpolated onto the particle at a 136 specified mixing rate

$$
\Omega_{m}=C_{M} \frac{\widetilde{\Gamma}+\widetilde{\Gamma}_{T}}{\Delta^{2}}
$$

${ }_{137}$ with a model constant $C_{M}=15$ for all the species, where $\Delta$ is the LES filter ${ }_{138}$ width. The optimal value of $C_{M}$ is determined by a sensitivity study on the 139 statistics (see Appendix A).

\section{2.3. LES/PDF solver}

${ }_{141}$ The recently developed "NGA/HPDF" code [10] is implemented for LES/PDF

142 of piloted turbulent jet flames. In order to solve the LES transport equations

143 for mass and momentum, the low Mach-number, variable-density Navier-

${ }_{144}$ Stokes equation solver (the "NGA" code) [31] is applied on a structured 
grid in a cylindrical coordinate system $(x, r, \theta)$. The resolved velocity field $\widetilde{\boldsymbol{u}}$ in LES is computed with second-order accuracy in space and time, where the tilde " " denotes density-weighted filtering with the LES filter width $\Delta=(\Delta x \Delta r(r \Delta \theta))^{1 / 3}$, where $\Delta x, \Delta r$ and $\Delta \theta$ are the mesh spacing in axial, radial and azimuthal directions, respectively. The pressure Poisson equation is solved to enforce continuity. In LES, the eddy-viscosity and gradientdiffusion assumptions are employed for subgrid stresses and scalar fluxes, respectively. The model coefficients for the turbulent viscosity and diffusivity are evaluated by the Lagrangian dynamic sub-grid scale model [32]. The major resolved fields in LES are the resolved velocity $\widetilde{\boldsymbol{u}}(\boldsymbol{x}, t)$, the density $\bar{\rho}(\boldsymbol{x}, t)$ and the turbulent diffusivity $\widetilde{\Gamma}_{T}(\boldsymbol{x}, t)$, which are also used in the PDF calculations.

The hybrid mesh/particle, highly-scalable PDF code "HPDF" [33] is employed to evolve the position and composition of each particle by solving a set of stochastic differential equations with second-order accuracy in space and time. In the present PDF calculations, nominally $N_{p c}=25$ particles are used in each cell. This is less than $N_{p c}=40 \sim 50$ typically used in previous LES/PDF studies [33, 34], because the statistical error with smoothing on a length scale greater than the grid size is demonstrated to scale as $N_{\text {tot }}^{-1 / 2}$, where $N_{t o t}$ is the total number of particles as the product of $N_{p c} \sim O(10)$ and the number of LES cells [16], whereas the error only scales as $N_{p c}^{-1 / 2}$ without smoothing. Our sensitivity studies also validate that the results with $N_{p c}=25$ are almost identical to those with $N_{p c}=40$ (not shown). The 
39-species DME/air skeletal mechanism [35] is adopted for the computation of combustion chemistry, and the in situ adaptive tabulation (ISAT) algorithm is applied to accelerate the time integration of the reaction rate $\boldsymbol{S}(\boldsymbol{\phi})$ in Eq. (5) or (8) [36, 37].

The Monte Carlo PDF particle solver and the finite-volume LES solver are fully coupled based on the transported specific volume approach [38]. An auxiliary scalar transport equation for the resolved specific volume $\widetilde{v}$, the inverse of $\bar{\rho}$, is evolved by the LES solver with a third-order bounded QUICK scheme [31]. This two-way coupling algorithm is verified by examining the consistency of the mean densities from the LES and PDF solvers in Appendix B.

The relative computational costs of three IEM implementations are summarized in Table 2. The IEM-MD calculation involves the evaluation of the mean-drift term $\widetilde{\mathcal{D}}_{\alpha}$ from $n_{\phi}$ resolved compositions, which adds $30 \%$ computational cost more than that with IEM-RW. Compared with IEM-MD, the IEM-DD calculation adds the evaluation of mixture-averaged transport properties $\Gamma_{(\alpha)}$ and the correction velocity $\boldsymbol{V}_{c}$, and its total computational cost is $40 \%$ more than that of IEM-RW.

\subsection{Grids and boundary conditions}

The computational domain is specified to be $L_{x} \times L_{r} \times L_{\theta}=60 D \times 30 D \times 2 \pi$ in the axial, radial and azimuthal directions. Three non-uniform structured grids are tested to determine if the LES grid resolution is adequate. The grid 
192

sizes are $96 \times 64 \times 16,128 \times 72 \times 32$, and $192 \times 108 \times 64$ in the axial, radial, and azimuthal directions with the grid points at

$$
x_{i}=L_{x} \frac{e^{i \alpha_{x} / N_{x}}-1}{e^{\alpha_{x}}-1}, \quad i=0, \ldots, N_{x}
$$

$$
r_{j}=L_{r} \frac{e^{j \alpha_{r} / N_{r}}-1}{e^{\alpha_{r}}-1}, \quad j=0, \ldots, N_{r},
$$

where $\alpha_{x}=\log \beta_{x} /\left(1-1 / N_{x}\right)$ and $\alpha_{r}=\log \beta_{r} /\left(1-1 / N_{r}\right)$ with the stretching ratios $\beta_{x}=2.0$ and $\beta_{r}=6.0$ in the axial and radial directions, respectively. In the grid sensitivity study in Appendix C, the statistical results from two fine grids $128 \times 72 \times 32$ and $192 \times 108 \times 64$ are almost converged, so the grid $128 \times 72 \times 32$ is utilized in further calculations. The average grid size within the flame zone is around $1.5 \mathrm{~mm}$ at $x / D=20$.

A separate pipe flow simulation is performed to generate the velocity inflow boundary conditions at the jet inlet. The pilot and coflow streams are represented as laminar with different bulk velocities to match those of the experiments. The mole fractions of inlet compositions in the jet, pilot, and coflow streams are the same as those in experiments [4]. The convective condition is used for the velocity and scalars at the outflow boundary [31] in the streamwise direction. The Dirichlet boundary condition is specified at the outer boundary in the radial direction. The stretched grid in the radial direction moves the transverse boundary far enough from the central turbulent jet, which can avoid the artifact of the Dirichlet boundary condition on entrainment near the jet. 


\section{Results and discussion}

The LES/PDF calculations of two piloted partially-premixed turbulent DME jet flames are performed. The simulation results for DME-D and DMEF flames are presented and discussed. The statistics are collected by time averaging over 15 flow-through times $T_{j}=L_{x} / U_{j}$ after the jet flame reaches a statistically steady state. The total CPU time of a LES/PDF run with IEM-MD model is around four days on 192 Intel Xeon E5 processor cores on the TH-2A supercomputer in Guangzhou, China (i.e., less than 20,000 core hours). The PDF and LES parts account for about $85 \%$ and $15 \%$ of the computational cost, respectively.

\subsection{Mean profiles}

In non-premixed combustion, the mixture fraction is essential to characterize mixing. The definition of the mixture fraction in the present study is the same as that in the DME flame experiments $[4,23]$. It is based on the adapted Bilger's formulation as

$$
\xi=\frac{2\left(Y_{C}^{*}-Y_{C, 2}\right) / w_{C}+\left(Y_{H}^{*}-Y_{H, 2}\right) / 2 w_{H}}{2\left(Y_{C, 1}-Y_{C, 2}\right) / w_{C}+\left(Y_{H, 1}-Y_{H, 2}\right) / 2 w_{H}} .
$$

Here, the adapted mass fractions $Y_{C}^{*}$ and $Y_{H}^{*}$, which are slightly different from the ordinary elemental mass fractions $Y_{C}$ and $Y_{H}$, are introduced in experimental data post-processing [23], considering that species concentrations of some intermediate hydrocarbons are not available from practical Raman/Rayleigh measurements. Moreover, $w_{C}, w_{H}$ are atomic masses, and 
subscripts 1 and 2 refer to the inflow conditions of the central jet and coflow, respectively.

For quantitative studies, we first compare the radial profiles at four axial locations $x / D=5,10,20$ and 40 in the experiment and LES/PDF. The first row of Fig. 1 shows the mean and the resolved root-mean-square (rms) mixture fraction $\xi^{\prime \prime}=\left(\left\langle(\widetilde{\xi})^{2}\right\rangle-\langle\widetilde{\xi}\rangle^{2}\right)^{1 / 2}$ from the experiment and from LES/PDF using IEM-RW and IEM-MD models for flame DME-D. Here angled brackets denote time averages. It is found that the results from the IEM-DD model are very close to those from IEM-MD, so they are omitted for clarity. The mean mixture fraction obtained by the IEM-MD model is in excellent agreement with the experimental measurements, but the IEM-RW model underpredicts the mean mixture fraction, slightly at $x / D=20$ and significantly at $x / D=40$ in the centerline region. In addition, the rms mixture fraction obtained by the IEM-MD model generally agrees well with the experimental data, whereas the rms from the IEM-RW model shows a slight over-prediction near the maximum rms mixture fraction at $x / D=5$ and in the centerline region at $x / D=10$. The discrepancies between the results from IEM-RW and IEM-MD are explained and discussed in detail in section 3.3.

For flame DME-F, the mean and rms mixture fraction profiles from the IEM-MD model, shown in the third row of Fig. 1, also generally agree well with the experimental data, and they are only slightly underestimated at $x / D=40$. In contrast, with the IEM-RW model the mean and rms mixture 

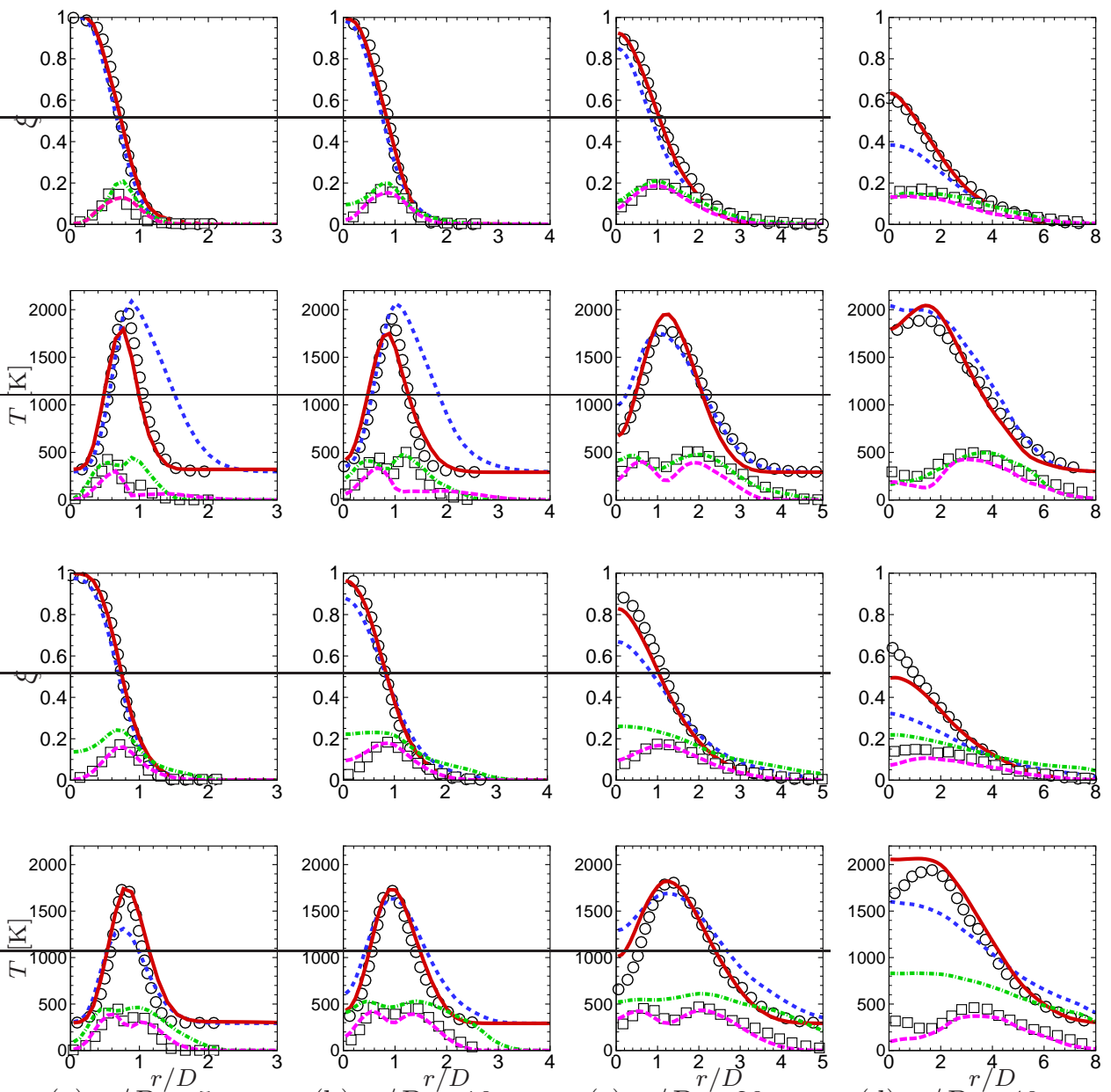

(a) $x / D=5$

(b) $x / D=10$

(c) $x / D=20$

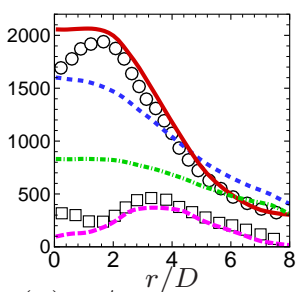

Figure 1: Mean and rms profiles of $\xi$ and $T$ at $x / D=5,10,20$ and 40 from experiment and LES/PDF for DME-D (upper two rows) and DME-F (bottom two rows) flames ( in experiment; dashed lines: mean in IEM-RW; solid lines: mean in IEM-MD; $\square$ : rms in experiment; dash-dotted lines: rms in IEM-RW; long-dashed lines: rms in IEM-MD). 
fractions are notably underpredicted and overpredicted, respectively. The discrepancies, in particular, those close to the centerline region, are partially attributed to the faster jet spreading rate, which appears to be caused by the random-walk representation of molecular transport in the particle transport equation in the IEM-RW model.

Compared with the temperatures from the experiment, the IEM-RW model overpredicts the mean temperature for DME-D, and also yields a wider distribution for DME-F. On the other hand, the IEM-MD model exhibits good agreement with the experiment, except for slight underpredictions of temperature at $x / D=5$ and 10 for DME-D and slightly overpredictions of temperature at $x / D=20$ and 40 for DME-F, as shown in the second and fourth rows of Fig. 1. In addition, the rms temperature from the IEM-MD model also shows much better agreement with the experimental data than that from the IEM-RW model for flame DME-F.

The mean mole fractions of the major species $\mathrm{CO}$ and $\mathrm{CO}_{2}$ are also compared in Fig. 2. The radial distributions of $\mathrm{X}_{\mathrm{CO}_{2}}$ are similar to temperature in Fig. 1 owing to the strong temperature dependency for $\mathrm{CO}_{2}$. The relatively accurate mean radial distributions of $X_{C O}$ are also achieved using IEM-MD, especially close to the centerline. In contrast, the calculations with IEMRW show some significant discrepancies compared to the experimental data. Furthermore, the $\mathrm{CO}$ prediction in the present LES/PDF is better than that obtained using the LES/flamelet-progress variable approach in [6], which can be caused by different modeling methods or different reaction mechanisms 

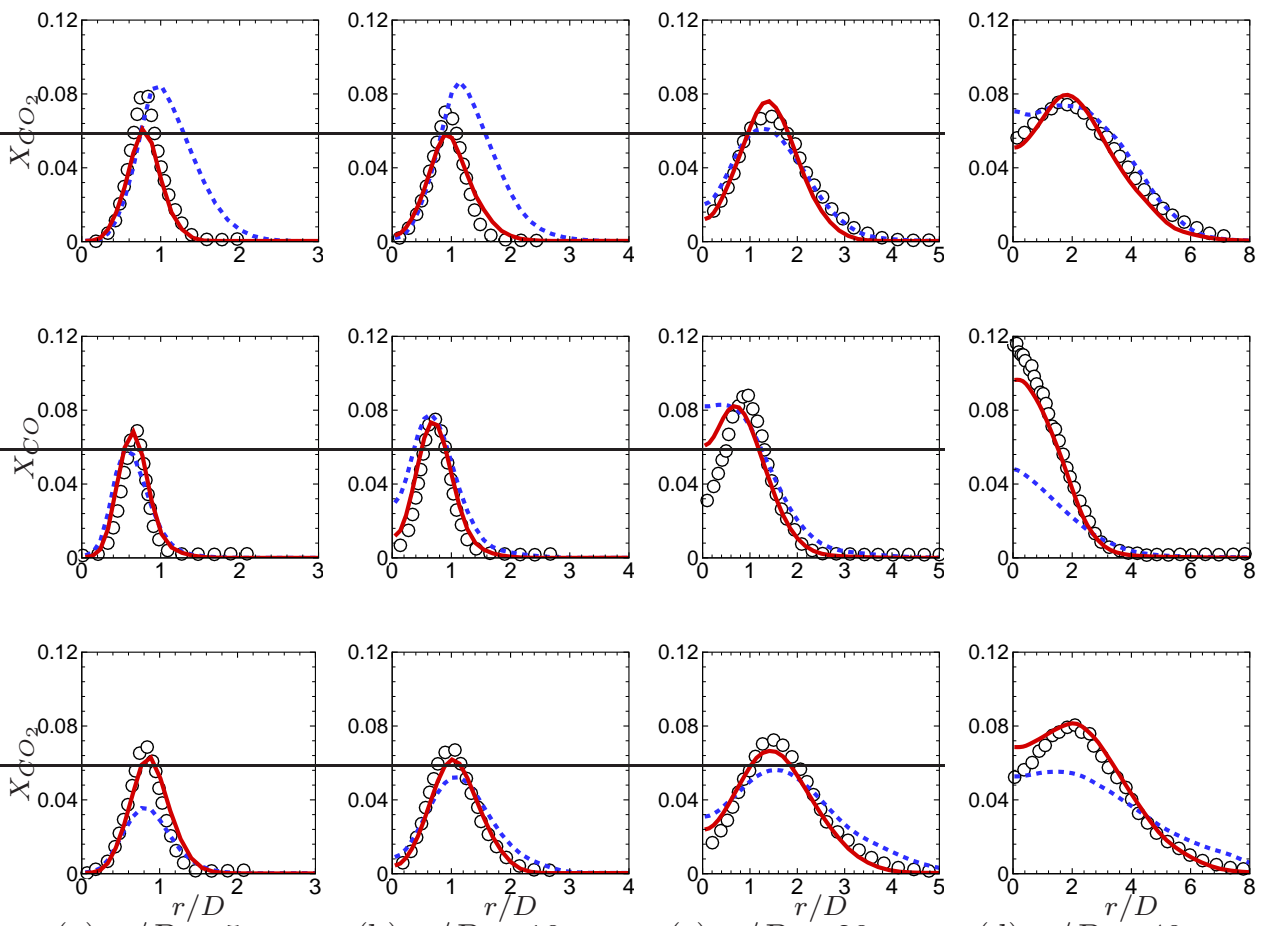

(a) $x / D=5$

(b) $x / D=10$

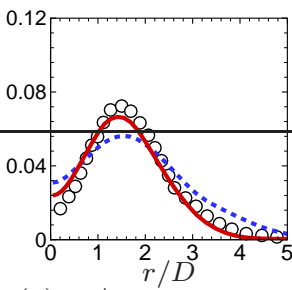

(c) $x / D=20$

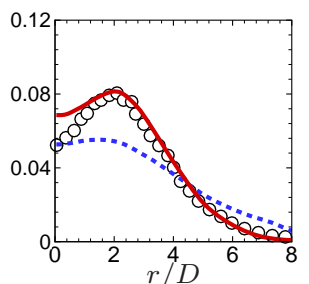

(d) $x / D=40$

Figure 2: Mean profiles of the mean mole fractions $X_{C O}$ and $X_{\mathrm{CO}_{2}}$ at locations $x / D=$ 5, 10, 20 and 40 from experiment and LES/PDF for DME-D (upper two rows) and DME-F (bottom two rows) flames ( $\circ$ : experiment; dash lines: IEM-RW; solid lines: IEM-MD).

used in these two studies.

\subsection{Conditional mean profiles}

278 In order to assess the combustion model for turbulence-chemistry interactions, averaged temperature and mole fraction of $\mathrm{CO}$ conditioned on 
the mixture fraction are compared between LES/PDF and experimental results in Fig. 3 for flame DME-D. The IEM-RW model overpredicts the conditional mean temperature around the stoichiometric mixture fraction at $x / D=10$ and 40 , and globally overpredicts the temperature by about $200 \mathrm{~K}$ at $x / D=40$. Moreover, the IEM-RW model significantly overpredicts $X_{C O}$ on the fuel-rich side at $x / D=10$ and underestimates $X_{C O}$ on the fuel-lean side at $x / D=40$, which are consistent with the results in Figs. 1 and 2. By comparison, the results from both IEM-MD and IEM-DD show very good agreement with the experimental data, but the difference is minor between these two IEM implementations.
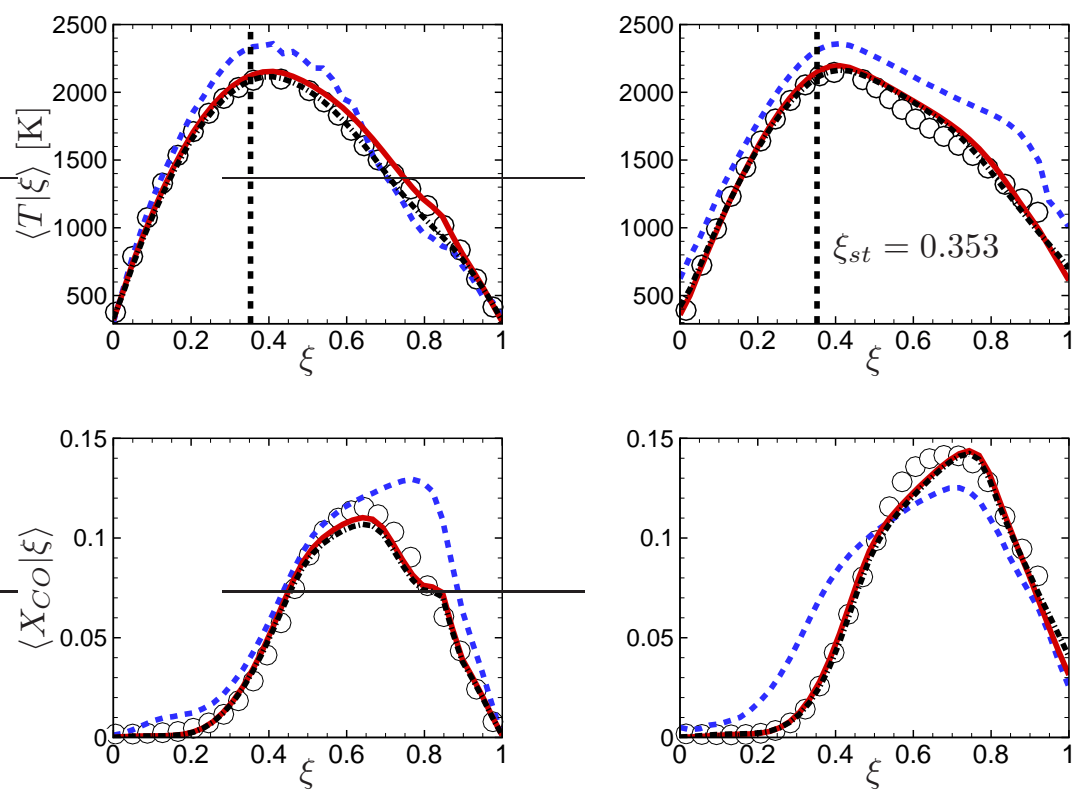

(a) $x / D=10$

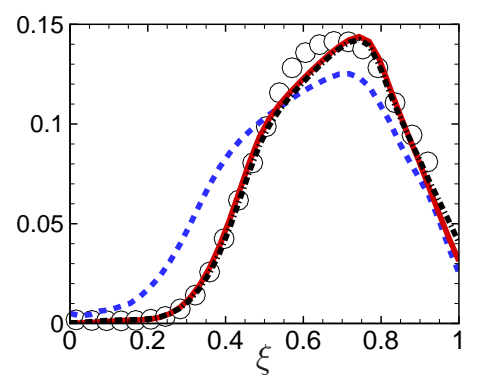

(b) $x / D=40$

Figure 3: Mean profiles of $T$ and $X_{C O}$ conditioned on $\xi$-space at axial locations $x / D=10$ and 40 from experiment and LES/PDF in DME-D flame (o: experiment; dashed lines: IEM-RW; solid lines: IEM-MD; dash-dotted lines: IEM-DD). 
Figure 4: Snapshots of the contour of $T$ (upper half) and $X_{O H}$ (lower half) on the $x-r$ plane cut from LES/PDF of DME-D and DME-F flames with the IEM-MD model.
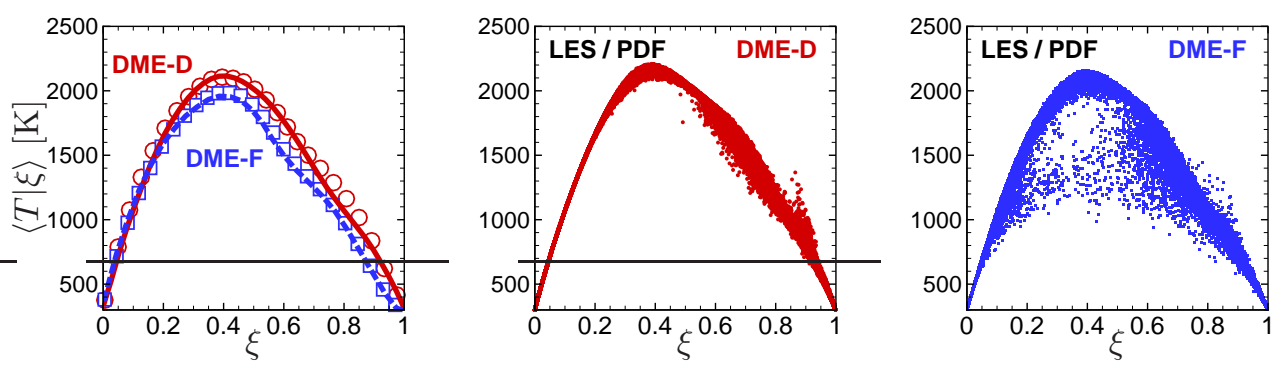

Figure 5: Mean profiles of $T$ (left) (symbols: experiment, lines: LES/PDF) and scatters of particle temperature conditioned on $\xi$-space from LES/PDF for DME-D (middle) and DME-F (right) flames with IEM-MD at $x / D=10$.

Snapshots of the instantaneous contours of temperature and mole fraction of $\mathrm{OH}$ for two flames are shown in Fig. 4. Flame DME-D shows continuous sheet-like structures without notable local extinctions, whereas DME-F ex- 
hibits extinction pockets (visible in the $\mathrm{OH}$ contours) that typically extend over several diameters of the jet and re-ignite further downstream. For quantitative comparisons between the two flames, conditional mean profiles and instantaneous scatter points of temperature from LES/PDF against the mixture fraction at $x / D=10$ are plotted in Fig. 5 . The mean profiles yield very good agreement with the experiment results in both flames. The maximum conditional mean temperature close to the stoichiometric mixture fraction in DME-D are approximately $100 \mathrm{~K}$ higher than that in DME-F, which also indicates more local extinctions in DME-F characterized by more scatter points of low temperatures than those in DME-D.

In general, the observations above suggest that IEM-MD is superior to IEM-RW in the model predictions in LES/PDF of DME jet flames. Additionally, the effect of differential diffusion is directly incorporated into the PDF equation in IEM-DD model, but the statistical results indicate that its effects are not significant in these two DME jet flames, which is consistent with the previous LES study using the flamelet/progress-variable model [6]. Detailed analysis about the differential diffusion is presented in section 3.4.

\subsection{Effects of molecular transport}

Previous LES studies of turbulent jet flames showed that the effect of molecular transport can be important [13] and the accurate representation of molecular transport is necessary in combustion LES models. The comparisons between the IEM-RW and IEM-MD model predictions in Figs. 1 and 
3 confirm that the accurate treatment of molecular transport is crucial in LES/PDF.

The averaged molecular diffusivities conditioned on temperature from LES/PDF in DME-D and DME-F flames at different locations $x / D=5,10,20$ and 40 are almost identical and are approximated by an empirical fit as

$$
\bar{\rho} \widetilde{\Gamma}=A+B\left(\frac{T}{T_{0}}\right)+C\left(\frac{T}{T_{0}}\right)^{2}
$$

where the constants $A=2.5 \times 10^{-5}, B=1.55 \times 10^{-5}, C=-4.5 \times 10^{-7}(\mathrm{~kg} / \mathrm{m}$. s) and the reference temperature is $T_{0}=292 \mathrm{~K}$. To assess the effects of molecular transport, we first compare the radial profiles of molecular diffusivity $\bar{\rho} \widetilde{\Gamma}$ and turbulent diffusivity $\bar{\rho} \widetilde{\Gamma}_{T}$ at different axial positions $x / D=10,20$ and 40 in Fig. 6. In the centerline region, the turbulent diffusivity is relatively small owing to the small grid size. For flame DME-D, the averaged molecular diffusivity is consistently larger than the turbulent diffusivity in the centerline region. Although the turbulent diffusivity increases further downstream with increasing turbulent intensity, the molecular diffusivity is still comparable to that of the turbulent diffusivity so its effect cannot be neglected. We remark that the molecular diffusivity particularly dominates over turbulent diffusivity on the centerline, and it continues to be dominant in the centerline region throughout the flow field. Thus the effect of molecular transport is expected to be significant in the centerline region, which is consistent with the results in Fig. 1. 


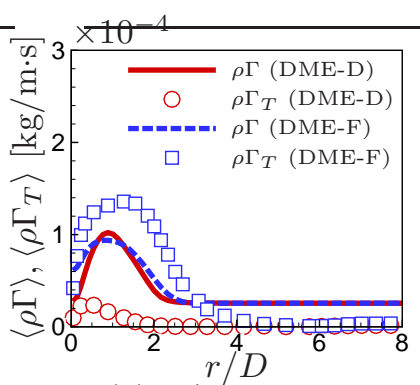

(a) $x / D=10$

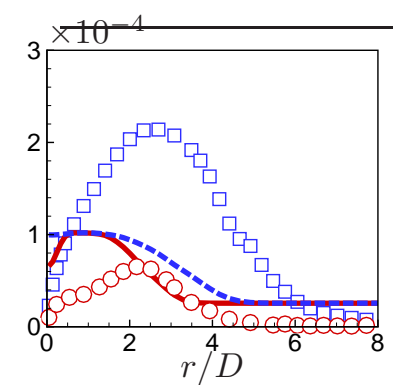

(b) $x / D=20$

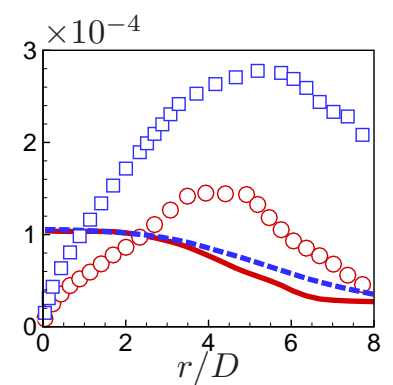

(c) $x / D=40$

Figure 6: Radial profiles of the molecular diffusivity and the turbulent diffusivity at $x / D=$ 10,20 and 40 for DME-D and DME-F flames (solid lines: molecular diffusivity in DME$\mathrm{D}$; ०: turbulent diffusivity in DME-D; dash lines: molecular diffusivity in DME-F; $\square$ : turbulent diffusivity in DME-F).

In order to illustrate the spatial distribution of the relative magnitude of the two viscosities, the contour plots of the time-averaged ratio and the instantaneous ratio of the turbulent viscosity to the molecular viscosity on the $x-r$ plane cut from LES/PDF of two flames are shown in Fig. 7. The peak time-averaged and instantaneous ratios are about 5 and 10 respectively for flame DME-D, and about 8 and 15 respectively for flame DME-F. The turbulent viscosity is significantly larger than the molecular viscosity in the shear layer between the central jet and pilot owing to the high turbulent intensity and potentially low grid resolution, and it is relatively small in the centerline region owing to the small grid size.

Moreover, the molecular diffusivity in DME-F is close to that in DME-D, while the turbulent diffusivity is much larger in DME-F than that in DME-D. Therefore, the effect of molecular transport is expected to be suppressed in DME-F compared with DME-D, which is partially supported by the results in Fig. 1. The discrepancies of temperature profiles at $x / D=5$ and 10 between 


$$
\frac{\partial}{\partial t} \widetilde{\phi_{\alpha}^{\prime \prime 2}}+\widetilde{\boldsymbol{u}} \cdot \widetilde{\nabla \phi_{\alpha}^{\prime \prime 2}}=\frac{1}{\bar{\rho}} \nabla \cdot\left(\bar{\rho} \widetilde{\Gamma}_{e} \nabla \widetilde{\phi_{\alpha}^{\prime \prime 2}}\right)+2 \widetilde{\Gamma}_{e}\left|\nabla \widetilde{\phi}_{\alpha}\right|^{2}-2 \Omega_{m} \widetilde{\phi_{\alpha}^{\prime \prime 2}}+2 \widetilde{\phi_{\alpha}^{\prime \prime} S_{\alpha}}
$$


8

the satisfies this criterion. In contrast, the second term on the right-hand-side of Eq. (15) for IEM-RW contains a nonvanishing molecular diffusivity in the DNS limit, so it yields the spurious production term $2 \widetilde{\Gamma}\left|\nabla \widetilde{\phi}_{\alpha}\right|^{2}$. As a result of this spurious production of fluctuations, the rms mixture fraction and temperature from IEM-RW are generally larger than those from IEM-MD in Fig. 1. Furthermore, the differences of the mean quantities between IEM-RW and IEM-MD may be due to indirect effects caused by the resulting increased composition fluctuations in the IEM-RW calculations.

\subsection{Differential diffusion}

Differential diffusion, in general, has a first-order effect on combustion processes $[20,22]$, and it can be characterized by the difference $\xi_{H}-\xi_{C}$, where $\xi_{H}$ and $\xi_{C}$ are the elementary mass fractions of the elements $\mathrm{H}$ and $\mathrm{C}$.

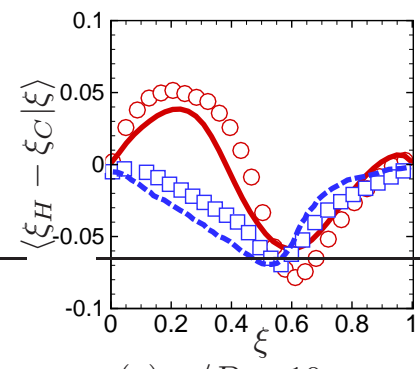

(a) $x / D=10$

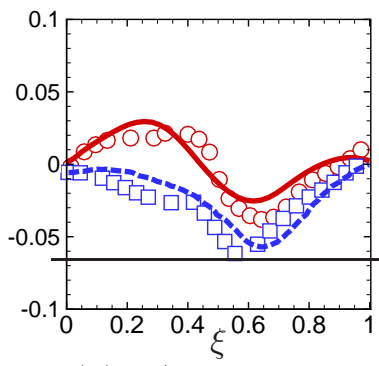

(b) $x / D=20$

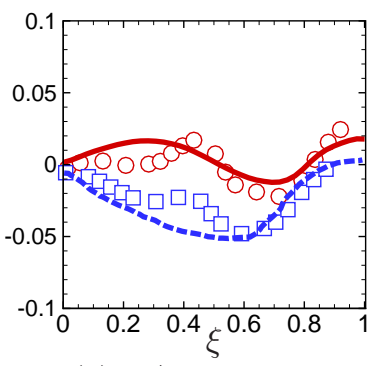

(c) $x / D=40$

Figure 8: Mean profiles of $\xi_{H}-\xi_{C}$ conditioned on $\xi$-space from experiment (circles: DMED, squares: DME-F) and LES/PDF (solid lines: DME-D, dashed lines: DME-F) with the IEM-DD model at $x / D=10,20$ and 40 .

This difference $\xi_{H}-\xi_{C}$ is non-zero only when different diffusivities are 
incorporated. Mean profiles of $\xi_{H}-\xi_{C}$ conditioned on mixture fraction at $x / D=10,20$ and 40 are shown in Fig. 8 for flame DME-D and DME-F. The results obtained using IEM-DD show overall good agreement with the experimental data, indicating that the differential diffusion effect is appropriately incorporated into the LES/PDF modeling. The decrease of the maximum difference implies that the influence of differential diffusion is diminished along the axial direction and the unity-Lewis-number assumption appears to be valid for the flames further downstream.

In PDF modeling, the effects of differential diffusion can be incorporated in mixing models through molecular transport, as in the present study, or through micro-mixing [40]. Although the effects of differential diffusion in Figs. 3 and 8 are not very significant in these DME jet flames, the implementation and validation of the IEM-DD model are still meaningful, because it can be directly utilized in turbulent premixed flames where differential diffusion can be significant. In such cases it would be interesting to explore whether the effects of differential diffusion are represented by molecular transport or micro-mixing at resolved or unresolved scales.

\section{Conclusions}

We report LES/PDF calculations of the piloted turbulent jet flames DME-D and DME-F with a skeletal chemical mechanism. The LES/PDF methodology is implemented using the highly-scalable NGA/HPDF code with three different models of molecular transport, namely IEM-RW, IEM- 
MD, and IEM-DD. The performance of the different models is analyzed through detailed a posteriori comparisons with the experimental data of the two target flames. The effects of molecular transport and differential diffusion are assessed.

In the LES of DME jet flames, molecular transport is comparable to turbulent transport near the jet exit and the centerline region, so it can play an important role in the evolution of the PDF equations. Better quantitative agreement for mean and rms mixture fraction, temperature and mole fractions of major species, and conditionally-averaged temperature is achieved with the IEM-MD model compared to the IEM-RW model.

Using the IEM-DD model, mean profiles of the difference between mixture fractions based on elementary mass fraction conditioned on mixture fraction yield good overall agreement with the experimental data. Hence, the differential diffusion is effectively implemented in LES/PDF, but it only has minor effects on the prediction of statistics of major quantities in this DME flame series.

Based on the successful implementations of IEM-MD and IEM-DD with detailed a posteriori validations in LES/PDF, it would be interesting to explore the performance of LES/PDF in turbulent premixed flames where differential diffusion may be more important than in partially-premixed jet flames. 


\section{Acknowledgments}

The authors thank F. Fuest and J. A. Sutton for sharing the experimental data. We also gratefully acknowledge Caltech, the University of Colorado Bolder and Stanford University for licensing the NGA code used in this work. This work has been supported in part by the National Natural Science Foundation of China (Grant Nos. 11521091 and 91541204) and the Thousand Young Talents Program of China, and the work at Cornell is supported by the U. S. Department of Energy Office of Science, Office of Basic Energy Sciences under Award Number DE-FG02-90ER14128.

\section{References}

\section{References}

[1] S. H. Park, C. S. Lee, Combustion performance and emission reduction characteristics of automotive DME engine system, Prog. Energy Combust. Sci. 39 (2013) 147-168.

[2] R. S. Barlow, A. Dreizler, J. H. Frank, J. Janicka, A. Kempf, R. P. Lindstedt, A. R. Masri, J. C. Oefelein, S. B. Pope, in: Ninth International Workshop on Measurement and Computation of Turbulent Nonpremixed Flames, July, 2010, Beijing, China, http://www.ca.sandia.gov/TNF/10thWorkshop/TNF10.html.

[3] B. Coriton, M. Zendehdel, S. Ukai, A. Kronenburg, O. T. Stein, S. K. Im, M. Gamba, J. H. Frank, Imaging measurements and LES-CMC 
modeling of a partially-premixed turbulent dimethyl ether/air jet flame, Proc. Combust. Inst. 35 (2015) 1251-1258.

[4] F. Fuest, G. Magnotti, R. S. Barlow, J. A. Sutton, Scalar structure of turbulent partially-premixed dimethyl ether/air jet flames, Proc. Combust. Inst. 35 (2015) 1235-1242.

[5] A. Bhagatwala, Z. Luo, H. Shen, J. A. Sutton, T. Lu, J. H. Chen, Numerical and experimental investigation of turbulent DME jet flames, Proc. Combust. Inst. 35 (2015) 1157-1166.

[6] S. Popp, F. Hunger, S. Hartl, D. Messig, B. Coriton, J. H. Frank, F. Fuest, C. Hasse, LES flamelet-progress variable modeling and measurements of a turbulent partially-premixed dimethyl ether jet flame, Combust. Flame 162 (2015) 3016-3029.

[7] S. B. Pope, PDF methods for turbulent reactive flows, Prog. Energy Combust. Sci. 11 (1985) 119-192.

[8] D. C. Haworth, Progress in probability density function methods for turbulent reacting flows, Prog. Energy Combust. Sci. 36 (2010) 168259.

[9] S. B. Pope, Small scales, many species and the manifold challenges of turbulent combustion, Proc. Combust. Inst. 34 (2013) 1-31.

[10] Y. Yang, H. Wang, S. B. Pope, J. H. Chen, Large-eddy simulation/PDF 
modeling of a non-premixed $\mathrm{CO} / \mathrm{H}_{2}$ temporally evolving jet flame, Proc. Combust. Inst. 34 (2013) 1241-1249.

[11] R. Cao, H. Wang, S. B. Pope, The effect of mixing models in PDF calculations of piloted jet flames, Proc. Combust. Inst. 31 (2007) 15431550.

[12] H. Wang, S. B. Pope, Lagrangian investigation of local extinction, reignition and auto-ignition in turbulent flames, Combust. Theor. Model. 12 (2008) 529-544.

[13] K. A. Kemenov, S. B. Pope, Molecular diffusion effects in LES of a piloted methane-air flame, Combust. Flame 158 (2011) 240-254.

[14] H. Wang, K. Kim, Effect of molecular transport on PDF modeling of turbulent non-premixed flames, Proc. Combust. Inst. 35 (2015) 11371145.

[15] R. McDermott, S. B. Pope, A particle formulation for treating differential diffusion in filtered density function methods, J. Comput. Phys. 226 (2007) 947-993.

[16] S. Viswanathan, H. Wang, S. B. Pope, Numerical implementation of mixing and molecular transport in LES/PDF studies of turbulent reacting flows, J. Comput. Phys. 230 (2011) 6916-6957.

[17] Y. Yang, S. B. Pope, J. H. Chen, An LES/PDF study of a turbulent 
lifted ethylene jet flame in a heated coflow, 8th U.S. National Combustion Meeting 20132 (2013) 913-920.

[18] W. Han, V. Raman, Z. Chen, LES/PDF modeling of autoignition in a lifted turbulent flame: Analysis of flame sensitivity to differential diffusion and scalar mixing time-scale, Combust. Flame 171 (2016) 69-86.

[19] S. Nambully, P. Domingo, V. Moureau, L. Vervisch, A filtered-laminarflame PDF sub-grid scale closure for LES of premixed turbulent flames. Part I: Formalism and application to a bluff-body burner with differential diffusion, Combust. Flame 161 (2014) 1756-1774.

[20] A. J. Aspden, M. S. Day, J. B. Bell, Lewis number effects in distributed flames, Proc. Combust. Inst. 33 (2011) 1473-1480.

[21] A. J. Aspden, M. S. Day, J. B. Bell, Characterization of low Lewis number flames, Proc. Combust. Inst. 33 (2011) 1463-1471.

[22] S. Lapointe, B. Savard, G. Blanquart, Differential diffusion effects, distributed burning, and local extinctions in high Karlovitz premixed flames, Combust. Flame 162 (2015) 3341-3355.

[23] F. Fuest, R. S. Barlow, J.-Y. Chen, A. Dreizler, Raman/Rayleigh scattering and CO-LIF measurements in laminar and turbulent jet flames of dimethyl ether, Combust. Flame 159 (2012) 2533-2562.

[24] S. B. Pope, Self-conditioned fields for large-eddy simulations of turbulent flows, J. Fluid Mech. 652 (2010) 139-169. 
[25] F. A. Jaberi, P. J. Colucci, S. James, P. Givi, S. B. Pope, Filtered mass density function for large-eddy simulation of turbulent reacting flows, J. Fluid Mech. 401 (1999) 85-121.

[26] J. Villermaux, J. C. Devillon, Représentation de la coalescence et de la redispersion des domaines de ségrégation dans un fluide par un modele d'nteraction phénoménologique, in: 2nd Int. Symp. on Chem. React. Eng., Elsevier, New York, 1972, pp. 1-13.

[27] C. Dopazo, E. E. O'Brien, An approach to the autoignition of a turbulent mixture, Acta Astronautica 1 (1974) 1239-1266.

[28] M. S. Anand, S. B. Pope, Diffusion behind line source in grid turbulence, Turbul. Shear Flows 4 (1985) 46-61.

[29] R. J. Kee, G. Dixon-Lewis, J. Warnatz, M. E. Coltrin, J. A. Miller, A fortran computer code package for the evaluation of gas-phase multicomponent transport properties, Tech. Rep. SAND86-8246, Sandia National Laboratories, 1986.

[30] R. J. Kee, F. M. Rupley, E. Meeks, J. A. Miller, CHEMKIN-III: A fortran chemical kinetic package for the analysis of gas-phase chemical and plasma kinetics, Tech. Rep. SAND96-8216, Sandia National Laboratories, 1996.

[31] O. Desjardins, G. Blanquart, G. Balarac, H. Pitsch, High order con- 
servative finite difference scheme for variable density low Mach number turbulent flows, J. Comput. Phys. 227 (2008) 7125-7159.

[32] C. Meneveau, T. S. Lund, W. H. Cabot, A lagrangian dynamic subgridscale model of turbulence, J. Fluid Mech. 319 (1996) 353-385.

[33] H. Wang, S. B. Pope, Large eddy simulation/probability density function modeling of a turbulent $\mathrm{CH}_{4} / \mathrm{H}_{2} / \mathrm{N}_{2}$ jet flame, Proc. Combust. Inst. 33 (2011) 1319-1330.

[34] V. Raman, H. Pitsch, A consistent LES/filtered-density function formulation for the simulation of turbulent flames with detailed chemistry, Proc. Combust. Inst. 31 (2007) 1711-1719.

[35] Z. Luo, T. Lu, J. Liu, A reduced mechanism for ethylene/methane mixtures with excessive NO enrichment, Combust. Flame 158 (2011) 12451254 .

[36] S. B. Pope, Computationally efficient implementation of combustion chemistry using in situ adaptive tabulation, Combust. Theor. Model. 1 (1997) 41-63.

[37] L. Lu, S. R. Lantz, Z. Ren, S. B. Pope, Computationally efficient implementation of combustion chemistry in parallel PDF calculations, J. Comput. Phys. 228 (2009) 5490-5525.

[38] P. P. Popov, H. Wang, S. B. Pope, Specific volume coupling and con- 
vergence properties in hybrid particle/finite volume algorithms for turbulent reactive flows, J. Comput. Phys. 294 (2015) 110-126.

[39] R. R. Tirunagari, S. B. Pope, LES/PDF for premixed combustion in the DNS limit, Combust. Theor. Model. (2016) 1-32.

[40] E. S. Richardson, J. H. Chen, Application of PDF mixing models to premixed flames with differential diffusion, Combust. Flame 159 (2012) $2398-2414$.

[41] D. H. Rowinski, S. B. Pope, Computational study of lean premixed turbulent flames using RANS-PDF and LES-PDF methods, Combust. Theor. Model. 17 (2013) 610-656.

[42] P. P. Popov, S. Pope, Large eddy simulation/probability density function simulations of bluff body stabilized flames, Combust. Flame 161 (2014) 3100-3133.

\section{Appendix A. Sensitivity study of $C_{M}$ in the IEM mixing model}

In PDF calculations, the scalar mixing rate is not known a priori and must be modeled. In the present study, a constant mechanical-to-scalar timescale ratio $C_{M}$ is applied in Eq. (10). This constant is a key model parameter and its value typically varies from 2 to $10[33,34]$, but larger values up to 100 were used some in previous LES/PDF calculations [41]. To determine the optimal value of $C_{M}$, the results of mixture fraction and 
temperature profiles with different values of $C_{M}$ are shown in Fig. 9. As may be seen, for flame DME-D and for flame DME-F at $x / D=5$ and 10 , there is little sensitivity to the value of $C_{M}$. Further downstream in flame DME-F, there is more sensitivity, and the value $C_{M}=15$ yields the best agreement with the experimental data. Consequently, this value $\left(C_{M}=15\right)$ is used in all of the other calculations reported.

\section{Appendix B. Consistency of the mean densities from the LES and PDF solvers}

In the LES/PDF context, the very different algorithms in the LES and PDF solvers pose challenges for the coupling of LES and PDF through the density or specific volume. The specific volume contains considerable random noise due to the nature of the Monte-Carlo PDF solution, so the feedback of the specific volume from the PDF solver to the LES pressure solver needs careful treatment. In the present study, the transported specific volume approach is employed, and this two-way coupling algorithm has been verified in several flames $[38,42]$. The radial profiles of mean density at different axial locations for DME-D flame with the IEM-MD model are shown in Fig. 10. We observe that the mean densities evaluated from the LES and PDF solvers are in good agreement with very slight discrepancy owing to the numerical errors involved in LES and PDF implementations [33]. 

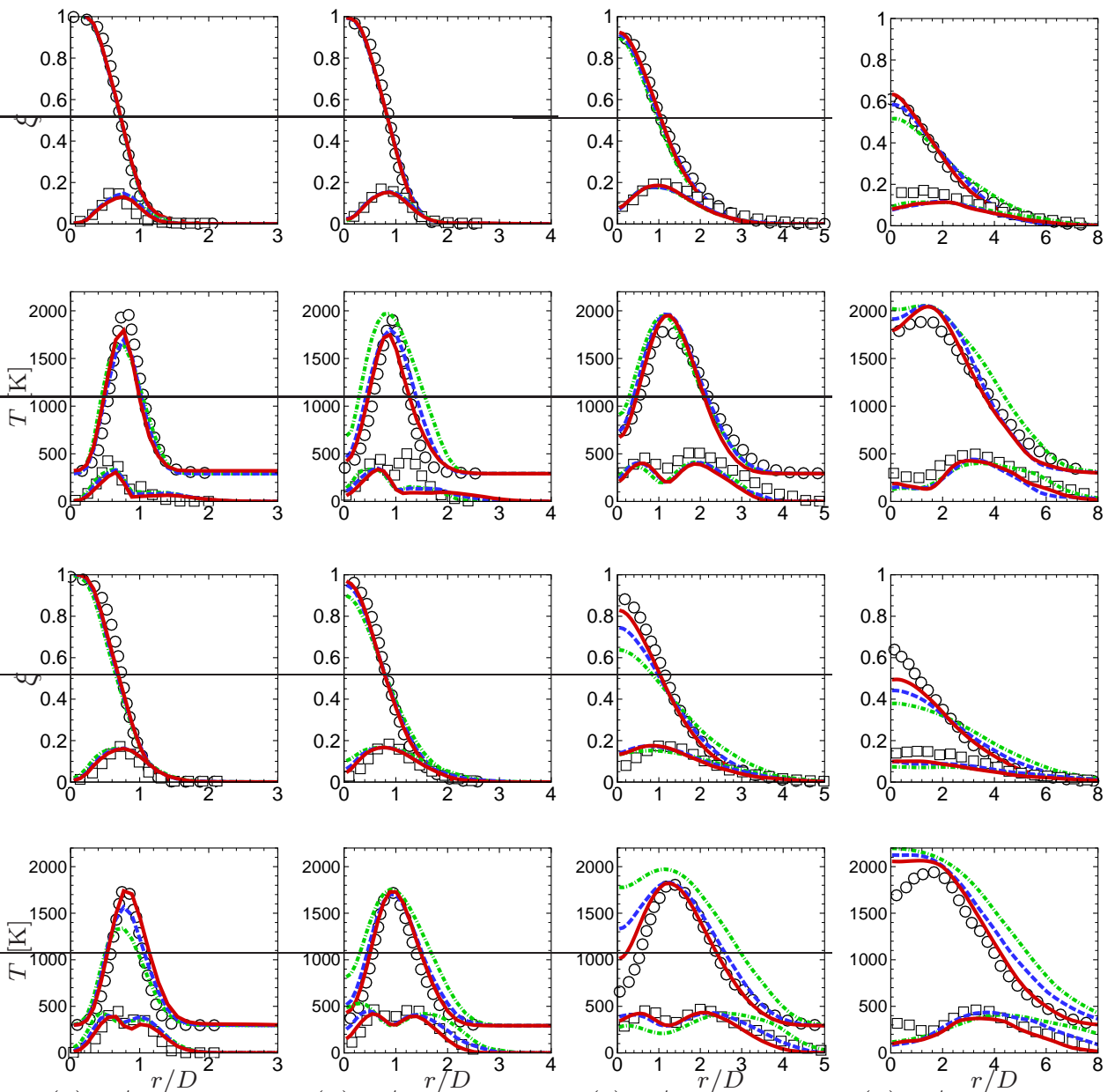
(a) $x / D=5$
(b) $x / D=10$

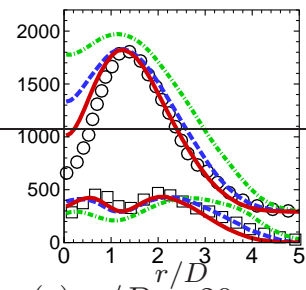

(c) $x / D=20$

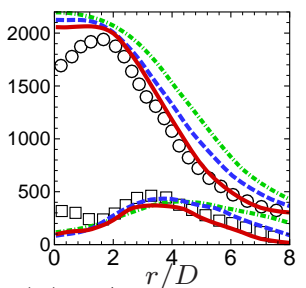

(d) $x / D=40$

Figure 9: Mean and rms profiles of $\xi$ and $T$ at locations $x / D=5,10,20$ and 40 from experiment and LES/PDF with IEM-MD model for DME-D (upper two rows) and DMEF (bottom two rows) flames ( $\circ$ : mean in experiment; $\square$ : rms in experiment; dash-dotted line: $C_{M}=5$; dashed line: $C_{M}=10$; solid line: $\left.C_{M}=15\right)$.

\section{Appendix C. Grid sensitivity study}

Three non-uniform structured grids are tested to determine if the LES grid resolution is adequate. The grid sizes are $96 \times 64 \times 16,128 \times 72 \times 32$, and $192 \times 108 \times 64$ in the axial, radial, and azimuthal directions. It is observed 


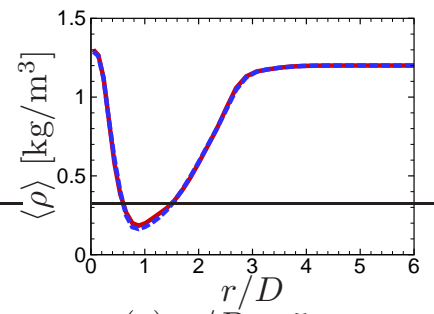

(a) $x / D=5$

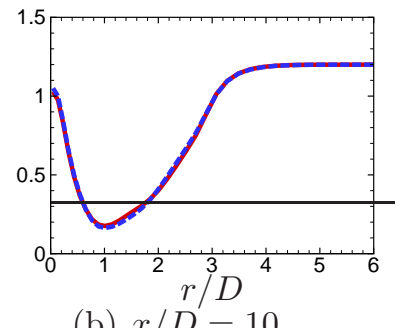

(b) $x / D=10$

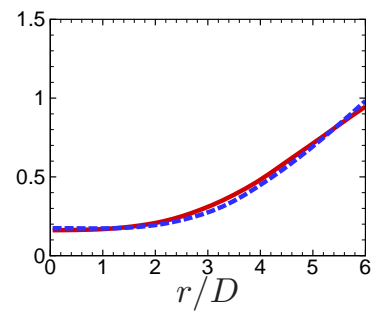

(c) $x / D=40$

Figure 10: Radial profiles of mean density at different axial locations $x / D=5,10$ and 40 for DME-D flame (solid lines: LES; dashed lines: PDF).

in Fig. 11 that the statistical results from the two finest grids $(128 \times 72 \times 32$ and $192 \times 108 \times 64)$ are almost converged. Thus, the grid $128 \times 72 \times 32$ is utilized in the other calculations reported in this paper. 

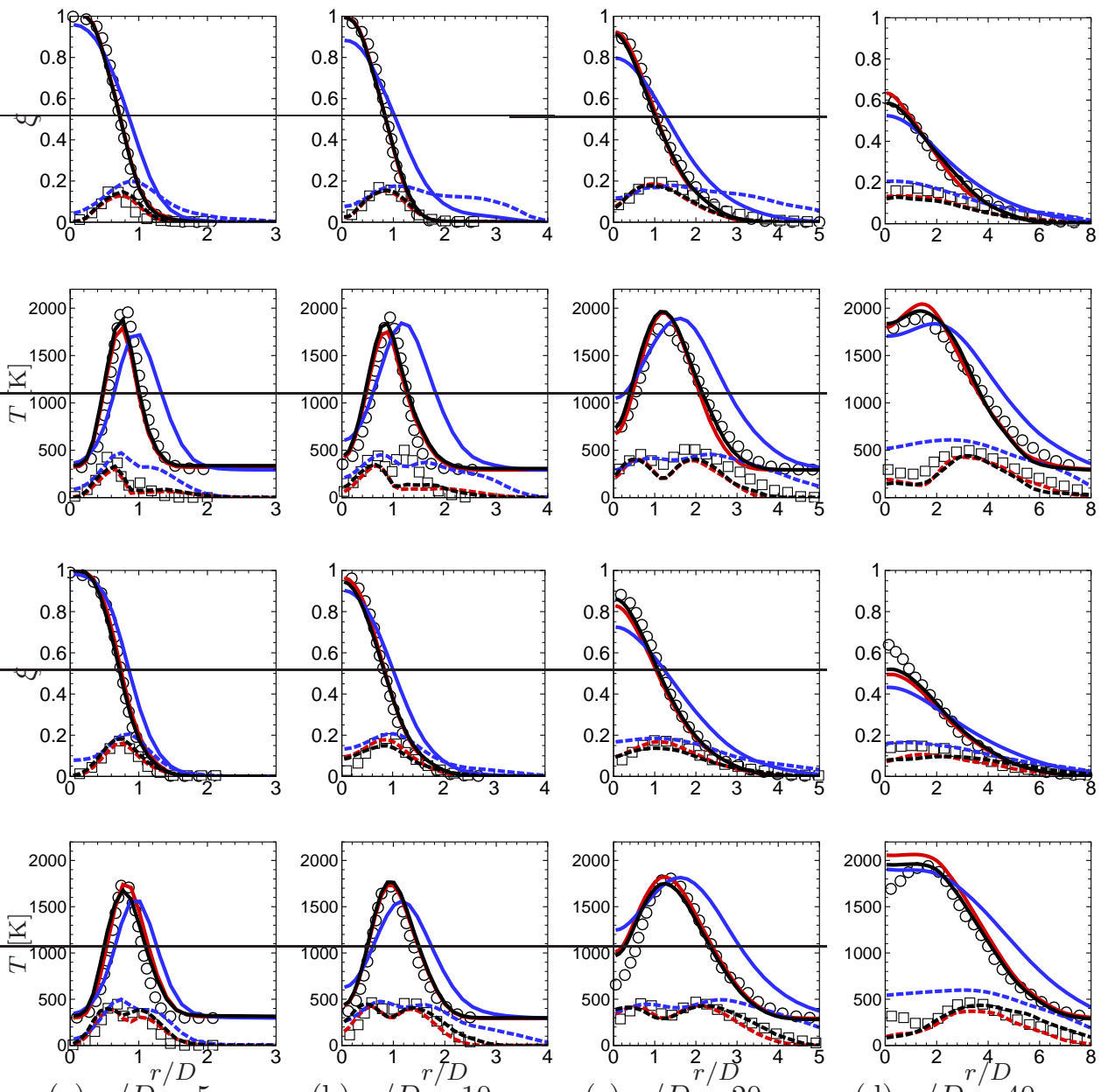

(a) $x / D=5$

(b) $x / D \stackrel{r / D}{=} 10$

(c) $x / D=20$

(d) $x / D=40$

Figure 11: Mean and rms profiles of $\xi$ and $T$ at locations $x / D=5,10,20$ and 40 from experiment and LES/PDF with IEM-MD model for DME-D (upper two rows) and DME$\mathrm{F}$ (bottom two rows) flames ( $\circ$ : mean in experiment; $\square$ : rms in experiment; blue lines: $96 \times 64 \times 16$ grid; red lines: $128 \times 72 \times 32$ grid; black lines: $192 \times 108 \times 64$ grid). (For interpretation of the references to color in this figure legend, the reader is referred to the web version of this article.) 